\title{
ESTUDIO DE LA INFANCIA EN LA RIOJA: ANÁLISIS DESDE UN ENFOQUE DE DERECHOS HUMANOS
}

\author{
Esther RAYA DIEZ \\ Profesora Titular de Trabajo Social y Servicios Sociales \\ UNIVERSIDAD DE LA RIOJA
}

Fermín NAVARIDAS NALDA

Profesor Titular de DidÁctica y ORganiZación ESCOLAR

UNIVERSIDAD DE LA RIOJA

Neus CAPARRÓS CIVERA

Profesora de Trabajo Social y Servicios Sociales

UNIVERSIDAD DE LA RIOJA

Domingo CARBONERO MUÑOZ

Profesor de Trabajo Social y Servicios Sociales

UNIVERSIDAD DE LA RIOJA

SUMARIO: I. Introducción. II. La infancia en la Rioja: datos y cifras. III. El derecho a un nivel de vida adecuado y la pobreza infantil. IV. Derecho a la salud. V. Derecho a la educación. VI. Protección y Prevención en la atención a la infancia. VII. El derecho a la participación. VIII. Recomendaciones principales.

RESUMEN: El presente artículo presenta los principales resultados del estudio sobre la situación de la infancia en la Rioja, tomando como punto de partida la Convención de los Derechos del Niño. El texto se estructura en ocho apartados, que presentan los aspectos más significativos de la situación de la infancia respecto al derecho implicado: nivel de vida; salud, educación y participación.

PALABRAS ClAVE: Infancia, derecho a un nivel de vida adecuado, derecho a la salud, derecho a la educación, derecho de participación

ABSTRACT: Using the Convention on the Rights of the Child, adopted by General Assembly the object of this article is the childhood in La Rioja. This article is divided into eight main parts, offering a compared view about the children in different Human Rights: standard of living, health, education and participation.

KEYwORDS: Children, the right to an adequate standard of living, the right to health, the right to education, the right to participation 


\section{Introducción}

La Convención sobre los Derechos del Niño es el tratado internacional que debe guiar las políticas, programas y servicios orientados a la infancia. Tomando como punto de partida el texto de la Convención junto a las Observaciones finales del Comité de los Derechos del Niño al Informe del Gobierno Español (octubre 20ı0) en las que el Comité presenta recomendaciones a partir de los informes elaborados por España ${ }^{\mathrm{I}}$ y los Informes sobre la Situación de la Infancia en España y en otras comunidades autónomas elaborados por UNICEF Comité Español desde 20Io, se elabora el I Informe de UNICEF sobre la Situación de la Infancia en La Rioja. Este informe ha sido elaborado en el marco de un convenio de colaboración entre la Universidad de La Rioja y UNICEF.

En el estudio se han consultado diferentes fuentes de información (estadísticas, memorias de actividad, normativas, etc.) procedentes de instituciones y organismos oficiales y de administraciones públicas que trabajan con la infancia. Esta información se ha complementado con la realización de una mesa de debate con personal técnico de diferentes organizaciones riojanas vinculadas a la atención a la infancia. También se ha consultado a los niños, a través de un cuestionario sobre aspectos relacionados con la Convención y con el derecho de participación.

En el artículo se presentan los aspectos más significativos de la situación de la infancia comenzando en el primer epígrafe con una presentación de los aspectos demográficos y normativos; en los siguientes epígrafes se presenta el análisis desde el enfoque de derechos humanos en cuanto al derecho a un nivel de vida adecuado y la situación de pobreza infantil; el derecho a la salud; el derecho a la educación y el derecho de participación, en una doble dimensión, por un lado, el derecho a dar la opinión y ser escuchado y el derecho de participación en la vida cultural y artística. Finalmente se presentan las principales conclusiones del trabajo.

\section{La infancia en la Rioja: datos y cifras}

La pirámide de población en La Rioja recoge las tasas reducidas de mortalidad y de natalidad propias de la segunda transición demográfica. La pirámide de población se define por el mayor tamaño de las cohortes del baby boom, situadas entre las franjas de edad de 35 a 55 años y el repunte de los nacimientos entre las edades de 3 a 7 años. Asimismo, en la franja de población menor de $\mathrm{I} 8$ años, se observa un retroceso de los nacimientos, ocasionado por el impacto de la crisis económica iniciada en el año 2008

Las migraciones y el envejecimiento se han tenido en cuenta en el estudio de los procesos demográficos en La Rioja. Un primer acercamiento a estas dos cuestiones se ha estudiado mediante el porcentaje de población extranjera y la tasa de dependencia entre los grupos de edad de -I8 años y de +65 años. El primer indicador muestra un mayor crecimiento migratorio en La Rioja en comparación con España, materializado en el mayor volumen de este grupo de población desde el año 2002 hasta el 2013.

\footnotetext{
${ }^{\mathrm{r}}$ https://www.unicef.es/sites/www.unicef.es/files/Observaciones.pdf
} 
A partir del cálculo de la tasa de dependencia de la población menor de i6 años ${ }^{2}$ y el índice de envejecimiento ${ }^{3}$ se ha analizado la estructura socio demográfica de La Rioja. Este análisis permite señalar que, por un lado, la tasa de dependencia se ha incrementado ligeramente entre el año 2000 (23,2\%) y el año 2013 (25,91\%), mientras que el índice de envejecimiento también ha aumentado entre los dos periodos de tiempo. Este hecho muestra el descenso de la población activa (+I6-64 años) respecto de la población menor de i6 años. Por otro lado, el incremento de la ratio de envejecimiento también se ha observado entre los años 2000 y 2013 , alcanzando $0,77 \%$ y $0,9 \%$ respectivamente 4 .

En La Rioja en 20II se aprobó el III Plan Integral de la Infancia 20II-20I4 con un presupuesto de $\mathrm{i} 86,6$ millones de euros para proteger, defender y atender a la infancia y a la adolescencia. El Plan aborda las necesidades de la población infantil y adolescente, su desarrollo personal y social y consta de cinco áreas de intervención: servicios sociales, deportes, educación, salud y justicia e interior. En total contiene 52 objetivos y I48 medidas.

Finalizamos este epígrafe referenciando la normativa en materia de infancia de la comunidad autónoma de la Riojas:

En primer lugar cabe citar la Ley I/2006, de 28 de febrero, de Protección de Menores de La Rioja. En la misma se regulan todas las competencias y potestades de la comunidad autónoma de La Rioja en materia de protección de menores y se atribuye a la Consejería competente en materia de servicios sociales una función directiva, que comprende el desarrollo de políticas públicas que promuevan el conocimiento y respeto de los derechos del menor en todos los ámbitos. En el artículo I.2 de la citada ley se entiende por protección de menores "el conjunto de actuaciones que deben realizar las Administraciones Públicas con la finalidad de promover el desarrollo integral de los menores, garantizar sus derechos, proporcionarles la asistencia moral o material de la que carezcan, total o parcialmente, en su medio familiar $y$, en su caso, procurar su reeducación y reintegración social".

El Decreto 3I/20II, de 29 de abril, aprueba la cartera de servicios y prestaciones del Sistema Público Riojano de Servicios Sociales explicitando la cartera de servicios. Tal como señala el Preámbulo, esta norma instrumentaliza uno de los ejes fundamentales de la Ley 7/2009, de Servicios Sociales, al reconocer como derechos subjetivos todos los servicios y prestaciones que recoge (49 servicios y iI prestaciones. Se trata, por tanto, de pasar de un sistema asistencial, en el que las ayudas estaban supeditadas a la existencia de disponibilidad presupuestaria, a un sistema garantista de reconocimiento de derechos a

\footnotetext{
${ }^{2}$ La tasa de dependencia "se define como el cociente entre la población residente en España a 1 de enero del año $t$ menor de 16 años o mayor de 64 entre la población de 16 a 64 años, expresado en tanto por cien. Se trata de un indicador con un claro significado económico, pues representa la medida relativa de la población potencialmente inactiva sobre la potencialmente activa". Fuente: http://www.ine.es/metodologia/t2o/metodologia_idb.pdf (I3 de Octubre de 20I4).

3 "Se define como el porcentaje que representa la población mayor de 64 años sobre la población menor de 16 años a 1 de enero del año t" Fuente: http://www.ine.es/metodologia/t2o/metodologia_idb.pdf (I3 de Octubre de 20I4)

${ }^{4}$ Las fuentes empleadas se corresponden con el padrón municipal a I de Enero de los años 2005, 2005, 2010 y $2013 \mathrm{http}: / /$ www.ine.es/jaxi/tabla.do?path=/t20/e245/po4/a2000/lo/\&file=000rioo2.px\&type=pcaxis

${ }^{5}$ Información recogida en la página web del Gobierno de la Rioja, en

https://www.larioja.org/npRioja/default/defaultpage.jsp?idtab=809406\&modelo=NA\&materiaP=I9o\&ma teriaS=080\&materia $\mathrm{T}=\mathrm{T}$
} 
todas las personas que cumplan los requisitos para acceder a los servicios y prestaciones que se establecen.

La cartera de servicios y prestaciones redunda en una mejora de las condiciones de vida de la población destinataria de las mismas. En la cartera se recogen específicamente los servicios y prestaciones dirigidos a los menores. Al final de este capítulo se presenta de forma resumida un cuadro con los principales servicios y prestaciones dirigidos a la infancia.

Junto a estas dos normativas, que podemos considerar centrales en la protección a la infancia, hay que citar un conjunto de decretos que aprueban los reglamentos de intervención y/u organización interna de los programas y servicios.

Además de las normativas de organización, hay que destacar la regulación de la concesión de prestaciones económicas. En este sentido, cabe destacar la Orden de 28 de octubre de 2004 por la que se regula la concesión de prestaciones económicas en el ámbito de la protección de la infancia que tiene por objeto establecer una serie de prestaciones económicas para la protección integral de los menores y la prevención, defensa y garantía de sus derechos. Estas prestaciones se articulan en dos modalidades: a) Ayudas económicas para paliar situaciones de riesgo de desprotección infantil y b) Prestaciones económicas a familias acogedoras.

A estas prestaciones específicamente dirigidas a la protección de la infancia se suman otras prestaciones que tienen por objeto atender las situaciones de exclusión social. En la Disposición Adicional del Decreto 28/20I4, de 27 de junio (BOR n ${ }^{\circ}$ 8I, de 2 de julio de 20I4) se establece un complemento de pobreza infantil en favor de los perceptores de las prestaciones de inserción social con menores a su cargo. Se trata de un complemento no consolidable, que se percibirá por una sola vez. Está dirigido a quienes perciban o hayan percibido Ingreso Mínimo de Inserción o Ayudas de Inclusión Social entre el I de julio y el 3I de diciembre de 20I4, y tengan menores de I8 años a su cargo. Asimismo hay que mencionar que se trata de un gasto estatal.

Finalmente, cabe considerar la Orden de ir de febrero de I998 de la Consejería de Salud, Consumo y Bienestar Social, por la que se crea el Consejo Sectorial de Infancia y Adolescencia. Se trata de un órgano de participación en materia de servicios sociales. En esta Orden se regula su composición y funcionamiento. Y en el artículo 6 se estable que se reunirá, como mínimo, una vez al semestre en sesión ordinaria. Como ha ocurrido con otros órganos similares, y a tenor, de la información recabada de los profesionales del sector, actualmente no está operativo, aunque se destaca la necesidad de espacios de coordinación en el sector de infancia y adolescencia.

\section{El derecho a un nivel de vida adecuado y la pobreza infantil}

En el Informe se ha hecho referencia al derecho a un nivel de vida adecuado. Los objetivos tratan de situar a la infancia como una etapa que condiciona el desarrollo del ciclo vital y el crecimiento de los niños. Siguiendo las recomendaciones de La Convención sobre los Derechos del Niño, la vulneración al desarrollo de sus capacidades conlleva a una privación del ejercicio de sus derechos. En concreto, el artículo 27 de la Convención sobre los Derechos del Niño (CDN) establece que "todo niño tiene derecho a beneficiarse de un nivel 
de vida adecuado para su desarrollo $y$ es responsabilidad primordial de padres y madres proporcionárselo. Es obligación del Estado adaptar medidas apropiadas para que dicha responsabilidad pueda ser asumida y que lo sea de hecho, si es necesario, mediante el pago de la pensión alimenticia." Por su parte el Comité de Derechos del Niño en sus Observaciones finales a España en el año 20Io recomienda al Estado que "formule políticas públicas para, en particular, abordar el problema de la pobreza infantil y elabore un plan nacional de lucha contra la pobreza infantil que establezca un marco coherente para determinar las acciones prioritarias contra la exclusión de los niños, con objetivos precisos y cuantificables, indicadores claros, plazos $y$ un apoyo económico $y$ financiero suficiente".

En este contexto, se trata de situar el nivel de vida en la Comunidad Autónoma de La Rioja (CAR), mediante la adaptación de un enfoque de nivel de vida. Además, se ha tratado de abordar aquellos factores que más influyen en la pobreza infantil. En concreto, se han abordado tres aspectos complementarios en este trabajo: I) la clasificación AROPE ${ }^{6}$ como indicador de las características económicas y de privación de los hogares riojanos; 2) la caracterización de la pobreza mediante la clasificación AROPE) y su vinculación a las características demográficas de los hogares ${ }^{7} .3$ ) la vinculación de los efectos de la política social en la reducción de la pobreza.

El alcance del trabajo y sus implicaciones se han visto condicionadas por la calidad de las fuentes de información utilizadas. En concreto, la encuesta de condiciones de vida (ECV) recoge datos relevantes a nivel de hogar y de individuos tales como el empleo, los ingresos o la composición demográfica de los hogares. La utilización de esta fuente de información es útil de cara a establecer comparaciones entre las condiciones de vida de los hogares españoles y riojanos. Sin embargo, el empleo de esta fuente de datos no deja de tener sus inconvenientes. En concreto, las cuestiones más importantes para realizar conclusiones respecto de la pobreza en la infancia, hacen referencia al escaso tamaño de la muestra y la mayor variabilidad de los resultados obtenidos en La CAR en comparación con otras comunidades autónomas.

Los principales resultados obtenidos, permiten señalar que la CAR en comparación al conjunto de España y de Comunidades Autónomas, presenta menores tasas de pobreza en el indicador AROPE y en sus distintos sub indicadores (la tasa de pobreza, individuos que viven en hogares de baja intensidad o de personas con problemas de privación material severa) $)^{8}$.

Los resultados obtenidos, han situado el mayor riesgo de los hogares con menores a cargo, respecto de cuestiones tales como el grado de dificultad para llegar a final de mes, no

\footnotetext{
${ }^{6}$ El indicador AROPE está compuesto de tres sub indicadores: el riesgo de pobreza después de las transferencias sociales, la carencia severa de bienes y los hogares que presentan baja intensidad de trabajo. A consecuencia de esta definición acordada en EUROSTAT, el indicador AROPE recoge a aquellas personas u hogares que se encuentran en alguno de los tres indicadores citados con anterioridad. Fuente: http://www.ine.es/ss/Satellite?L=es_ES\&c=INESeccion_C\&cid=I25994I637944\&p=I254735IIO672\&pagename =ProductosYServicios/PYSLayout (Consultado el I3 de octubre de 20I4)

${ }^{7}$ En base a la información proporcionada por el Instituto Riojano de Estadística, se ha contrastado la información de aquellos hogares formados por 2 adultos con niños y otros hogares con niños, en comparación a aquellos hogares sin niños (una persona sin niños, 2 adultos sin niños y otros hogares sin niños) y el conjunto total de los hogares de La CAR.

${ }^{8}$ La comparación entre La CAR y el conjunto de España, muestran resultados más bajos para en los indicadores de tasa AROPE (2I,2\% y $29.2 \%$ ), la tasa de pobreza (І6.6\% y $22.2 \%)$, la baja intensidad en el trabajo (I0,7\% y I7.I\%) y la privación material (2\% y 7.I\%)
} 
poder permitirse unas vacaciones al menos una semana al año, la proporción de hogares que no pueden permitirse una comida de carne, pollo o pescado cada dos días o la proporción de hogares que no pueden hacer frente a gastos imprevistos ${ }^{9}$.

En el estudio se comprueba el descenso del nivel de vida en el conjunto de España y en La CAR. A su vez, los resultados obtenidos en base a la ECV 20I4 muestran los mejores resultados de La CAR en referencia al indicador AROPE y a los diferentes sub indicadores. Los resultados obtenidos en La CAR han confirmado las hipótesis formuladas en anteriores trabajos en los que se afirmaba las mayores tasas de pobreza y de exclusión social en los hogares con niños a cargo. Los análisis han confirmado el escaso impacto de las políticas sociales para compensar las cargas de menores en el hogar y la importancia del empleo entre los miembros del hogar para escapar de la situación de la pobreza.

No obstante, hay que destacar que el estudio de las condiciones de vida de la infancia en la CAR, precisa de fuentes de datos más completas. En concreto, la ausencia de una muestra representativa para el conjunto de esta comunidad imposibilita la cuantificación y caracterización de la pobreza infantil. Algunas de las cuestiones más relevantes, se reflejan en la imposibilidad de caracterizar la composición demográfica (sexo, origen de procedencia, edad o estado civil), económica (tasa de pobreza infantil, tipo de ayudas recibidas o si el volumen de las cuantías de éstas permiten escapar de la pobreza a los menores) o laboral (situación laboral de los padres en los hogares con menores a cargo) de los hogares con cargas familiares y su vinculación a problemas de nivel de vida.

\section{Derecho a la salud}

La OMS establece que el goce del grado máximo de salud que se pueda lograr es uno de los derechos fundamentales de todo ser humano. Y que el derecho a la salud incluye el acceso a una atención sanitaria oportuna, aceptable, asequible y de calidad satisfactoria.

El derecho a la salud significa que los Estados deben crear las condiciones que permitan que todas las personas puedan vivir lo más saludablemente posible. El derecho a la salud no debe entenderse como el derecho a estar sano. Y que los problemas de salud suelen afectar en una proporción más alta a los grupos vulnerables y marginados de la sociedad.

El derecho a la salud significa que los gobiernos deben crear las condiciones que permitan a todas las personas vivir lo más saludablemente posible. Esas condiciones incluyen las disponibilidades garantizadas de servicios de salud, condiciones de trabajo

\footnotetext{
${ }^{9}$ En primer lugar, los hogares con mucha dificultad y alguna dificultad para llegar a fin de mes afecta en mayor medida a los hogares formados por 2 adultos con niños (I4.45\% y $24.71 \%$ )) y a otros hogares con niños (I $8,72 \%$ y $20.68 \%$ ), frente a aquellos hogares formados por dos adultos sin niños (І2,78\% y ı०.23\%) y a otros hogares sin niños (13,75 y I5.I2\%). En segundo lugar, los hogares con alguna dificultad para llegar a fin de mes, afectan en mayor medida los hogares. En cuanto a la imposibilidad de permitirse unas vacaciones al menos una semana al año, los grupos de población más afectados hacen referencia a aquellos hogares formados por 2 adultos con niños $(36,16 \%)$ y otros hogares con niños $(39,28 \%)$, frente a los hogares formados por dos adultos $\sin$ niños $(26,94 \%)$ y otros hogares sin niños (3I,97\%). En tercer lugar, la proporción de hogares que no pueden permitirse una comida de carene, pollo o pescado cada dos días afecta con mayor intensidad a los hogares formados por otros hogares con niños (3,79\%), que a los hogares formados por 2 adultos sin niños (I,29\%) y otros hogares sin niños $(2,73 \%)$. Finalmente, los hogares que no pueden hacer frente a imprevistos afectan en mayor medida a los hogares formados por 2 adultos sin niños $(29,73 \%)$ y otros hogares sin niños $(39,28 \% \%)$, que a aquellos hogares formados por dos adultos sin niños $(26,94 \%)$ y otros hogares sin niños (3I,97\%).
} 
saludables y seguras, vivienda adecuada y alimentos nutritivos. Un derecho a la salud que está consagrado en tratados internacionales y regionales de derechos humanos y en las constituciones de países de todo el mundo. (OMS, 20I5)

En esta línea encontramos la Convención de los Derechos de los Niños, primera ley internacional sobre los derechos de los niños, de carácter obligatorio para los Estados firmantes, en la que se reconoce, a lo largo de los 54 artículos, los diferentes derechos de los niños y niñas de entre los cuales está el de salud.

Varios son los artículos que hacen mención a este derecho, pero el artículo que aborda directamente el tema de la salud es el artículo 24 en el que se hace referencia explícita al derecho que tienen los menores a disfrutar del más alto nivel posible de salud $y$ a tener acceso a servicios médicos $\gamma$ de rehabilitación, con especial énfasis en aquéllos relacionados con la atención primaria de salud, los cuidados preventivos y la disminución de la mortalidad infantil. El Estado miembro firmante tiene la responsabilidad de tomar las medidas necesarias, orientadas a la abolición de las prácticas tradicionales que puedan ser perjudiciales para la salud del menor.

Igualmente el Comité de los Derechos del Niño en la Observación General n ${ }^{\circ}$ I5, recoge la importancia de seguir velando por la salud infantil desde la óptica de los derechos del niño, en el sentido de que todos tienen que tener el derecho a las oportunidades de supervivencia, crecimiento y desarrollo en un contexto de bienestar físico, emocional y social al máximo de sus posibilidades y vivir en condiciones que le permitan disfrutar del más alto nivel posible de salud, mediante la ejecución de programas centrados en los factores subyacentes que determinan la salud.

La Comunidad autónoma de La Rioja siguiendo las directrices marcadas por la Observación General y la Convención de los derechos de los niños diseña el III Plan Integral de la Infancia 20II-20I4 que sienta las bases para atender a la infancia de la Comunidad riojana en sus diferentes dimensiones, siendo uno de ellas la de salud, por lo que se va a seguir trabajando para conseguir un estado de salud que permita el crecimiento y desarrollo óptimo del niño en todas sus vertientes.

Para poder saber el estado de salud la CAR ha diseñado planes propios y recogido los datos que le llevan a promover recursos y servicios para favorecer la salud de los menores.

No obstante la realización del Informe sobre la situación de la infancia en La Rioja ha puesto de manifiesto algunas necesidades que aun estando en vías de ser cubiertas siguen requiriendo intervención.

Los principales problemas de salud detectados en la infancia y la adolescencia son la obesidad, trastornos de comportamiento siendo el más frecuente el déficit de atención y la hiperactividad, los trastornos de comportamiento alimentario (anorexia y bulimia), el consumo de tabaco, alcohol y otras drogas, el maltrato y las desigualdades socio sanitarias.

Para dar respuesta existen dispositivos, recursos y servicios, pero hay que seguir trabajando en programas de prevención y de promoción de la salud, dotar de más

\footnotetext{
${ }^{\text {Io }}$ Recuperado de http://www.who.int/mediacentre/factsheets/fs323/es/ el 29 de octubre de 2015
} 
profesionales en atención sobre todo en temas de salud mental, tanto de patología como de trastornos y seguir promocionando hábitos de vida saludables relacionados no solo con la alimentación, sino con el consumo de tabaco, alcohol o cualquier otra sustancia.

\section{Derecho a la educación}

La educación es un derecho humano fundamental y un bien público irrenunciable para poder ejercitar todos los demás derechos. La Convención sobre los Derechos del Niño (UNESCO, 2006) en sus artículos 28 y 29 concibe la calidad de la educación como un proceso permanente orientado a garantizar los máximos niveles de desarrollo y aprendizaje posibles en el niño, a fin de prepararlo para una vida adulta activa, inculcarle el respeto de los derechos humanos elementales y desarrollar su respeto por los valores culturales y nacionales propios y de civilizaciones distintas a la suya.

Desde este enfoque de derechos, evaluar la calidad de la educación supone atender a cinco criterios básicos (UNESCO, 2007): equidad, pertinencia, relevancia, eficacia y eficiencia. La equidad comprende los principios de igualdad y diferenciación, ya que tan sólo una educación ajustada a las necesidades de cada uno asegurará que todas las personas tengan las mismas oportunidades de hacer efectivos sus derechos y alcanzar los fines de la educación en condiciones de igualdad. La pertinencia alude a la necesidad de flexibilizar la enseñanza para que la educación dé respuesta a la diversidad de necesidades de los individuos y contextos. La relevancia se refiere al desarrollo de las competencias necesarias para participar en los diferentes ámbitos de la vida humana y construir proyectos de vida con relación a los otros. La eficacia y la eficiencia son atributos de la acción pública que nos indican en qué medida se alcanzan los objetivos y se usan adecuadamente los recursos destinados a esta tarea. De acuerdo con todo ello, presentamos a continuación un resumen de los datos más relevantes sobre el derecho a la educación en la Comunidad Autónoma de La Rioja.

Con relación a la escolarización, se confirma la universalización de la educación obligatoria, extendiéndose de forma comprensiva a la totalidad de la población escolar analizada durante el curso 20I4/15. Centrando el análisis en las etapas de enseñanza no obligatoria, en Educación Infantil (o-6 años) son más elevadas las tasas de escolarización asociadas al segundo ciclo (entre el 94,9\% y casi el 96\%) que las del primer ciclo (entre un $24,6 \%$ a los 2 años y un 5,4\% con menos de un año). Adentrándonos en el primer ciclo de esta etapa (o a 3 años), resulta significativo el alto porcentaje de escolarización en centros privados no concertados en relación con el total de alumnos matriculados (51,25\%). Dada la importancia que posee esta primera etapa para el desarrollo y el aprendizaje futuro del niño, así como para mejorar la equidad de los resultados y los niveles globales de competencias adquiridos (UNICEF, 2008) ${ }^{\mathrm{II}}$, parece aconsejable desarrollar políticas encaminadas a incrementar de forma significativa la tasa neta de escolarización durante este primer periodo de la Educación Infantil en La Rioja.

Otro aspecto a destacar con relación a la escolarización y el entorno educativo de La Rioja es la gran diversidad y riqueza multicultural que caracteriza a los centros escolares de

${ }^{\text {II }}$ UNICEF (2008). El cuidado infantil en los países industrializados: transición y cambio. Report Card Innocenti $\mathrm{N}^{\circ}$ 8. Centro de Investigaciones Innocenti de UNICEF, Florencia. 
esta Comunidad. Considerando el peso del alumnado extranjero matriculado en las enseñanzas no universitarias en cada comunidad autónoma de España sobre el total de su alumnado, el mayor porcentaje se observa en La Rioja $(\mathrm{I} 4,4 \%)^{\mathrm{I} 2}$ muy por encima de la media de España (9,9\%). Los datos analizados invitan a desarrollar un modelo de educación inclusiva que dé respuestas pertinentes a toda la diversidad de necesidades (por ejemplo, aquellas derivadas del conocimiento del idioma, la escolarización previa, la situación económica o sociocultural de la familia) que resultan susceptibles de convertirse en barreras de aprendizaje y afectar a los logros académicos.

Respecto a los resultados de aprendizaje, La Rioja obtiene un buen rendimiento en las competencias de matemáticas y en ciencias, significativamente superiores en ambos casos al promedio de la OCDE, la UE y a los de España en su conjunto. Sin embargo, el rendimiento en la competencia lectora es significativamente menor que en el caso de las matemáticas y las ciencias. Sobre la base de los datos proporcionados por PISA (20I2), el índice social, económico y cultural familiar (ISEC) es un factor determinante a la hora de explicar algunas de estas diferencias de rendimiento académico encontradas ${ }^{13}$. En España, el ISEC de las familias de los alumnos equivale a -0,I9, inferior al promedio que establece la OCDE $(\circ, \circ)$ con una desviación típica de I,O०. En el caso concreto de La Rioja el ISEC equivale a -O,I5 puntos, muy próximo a la media de España $(-0,19)$ e inferior al promedio de la UE $(0,2)$. Tras el análisis efectuado, se observan valores medios en las competencias investigadas mejores a los que cabría esperar en función de su ISEC medio (-o,I5), destacando en mayor medida el valor obtenido en la Competencia Matemática. No obstante, y a la vista de los resultados de rendimiento obtenidos en los últimos años en función del ISEC (PISA 2006 y PISA 2009), parece prioritario hacer un mayor esfuerzo en mejorar las condiciones y los recursos que resulten necesarios para aumentar el nivel de equidad en beneficio de la calidad del sistema educativo investigado, especialmente si se atiende al contexto socioeconómico y cultural menos favorecido de un alto porcentaje de alumnado extranjero matriculado en esta Comunidad.

Otro indicador relevante a tener en cuenta en el apartado relativo a los resultados educativos es el porcentaje de alumnado que repite curso en la etapa obligatoria ${ }^{\mathrm{I}}$, principalmente con el fin de establecer medidas orientadas a prevenir el fracaso escolar, reducir sustancialmente las tasas de abandono escolar temprano y favorecer la dinámica y el clima de convivencia en las aulas. Según los últimos datos estadísticos aportados por el Informe PISA 20I2, el 33,9\% de los alumnos de I5 años ha repetido curso en España. En el caso concreto de La Rioja este porcentaje es ligeramente superior, el 36,1\%. En la OCDE y UE las tasas de repetición son bastante más reducidas, el I $6,1 \%$ y el $15 \%$ respectivamente. A la vista de estos datos negativos, si se quiere alcanzar los objetivos de calidad establecidos a nivel europeo y reducir las tasas de fracaso escolar de los alumnos en La Rioja, es

\footnotetext{
${ }^{12}$ Fuente: Instituto Nacional de Evaluación Educativa. Sistema Estatal de Indicadores de la Educación 2014. Ministerio de Educación, Cultura y Deporte, 20I4, pág. 30.

${ }^{13}$ La relación entre este índice y el rendimiento de los estudiantes se suele interpretar como una medida de equidad de los sistemas educativos. El ISEC se considera un indicador de riqueza compuesto por tres medidas relacionadas con el nivel más alto de educación alcanzado por los padres, el prestigio de la profesión con mayor consideración social de los padres y el nivel de recursos domésticos. De esta forma, el sistema educativo de una comunidad se considera más equitativo, cuanto menor sea el impacto de la variación del ISEC del alumnado en su rendimiento.

${ }^{14}$ Véase en este sentido la reciente publicación del Instituto Nacional de Evaluación Educativa (20I4). Sistema estatal de indicadores de la educación. Edición 2014. Madrid: Ministerio de Educación, Cultura y Deporte.
} 
indispensable poner en práctica medidas inclusivas urgentes que den una respuesta lo más eficaz y eficiente posible a esta realidad educativa.

Con relación a la tasa de abandono temprano de la educación y la formación ${ }^{15}$ en el 2013 en La Rioja es del I8,2\%, un porcentaje que se ha visto reducido aproximadamente 4,6 puntos con respecto al año 2012 (22,8\%). Esta tendencia descendente en la evolución del abandono escolar temprano, ha supuesto que La Rioja deje de ocupar un lugar entre las comunidades con peores resultados en relación con este indicador importante de evaluación de los sistemas educativos, ya que en 20II mostraba un abandono superior a la media de España $(26,5 \%)$. No obstante, y pese al descenso notable de la tasa de abandono temprano, el resultado obtenido puede quedar algo empañado cuando se analiza la situación a nivel nacional y europeo, ya que entre los objetivos prioritarios de España y la propia Estrategia Europea 2020 se encuentra situar la tasa de abandono temprano por debajo del I5\% y Iо\% respectivamente (Instituto Nacional de Evaluación Educativa, 20I4). De ahí que sea necesario seguir insistiendo en reforzar y activar nuevas medidas específicas de atención a la diversidad educativa de alumnos que no han completado el nivel de la segunda etapa de la Educación Secundaria y no siguen ningún tipo de educaciónformación.

Para finalizar con este apartado, podemos concluir que esta caída notable del abandono temprano de la educación o formación en La Rioja guarda cierta relación con el aumento significativo de estudiantes que cursan enseñanzas relacionadas con la Formación Profesional en general. Así por ejemplo, en los últimos cinco cursos, el incremento en las matriculaciones de los ciclos formativos ha subido en un $27 \%$ aproximadamente. Una tendencia similar ocurre en el caso de los Programas de Cualificación Profesional Inicial (32\%) o en la Educación de Adultos y a Distancia (I5\%). Entre otras razones que pueden explicar este aumento significativo de matrícula en los estudios relacionados con la formación profesional, se encuentra la crisis económica como factor determinante de que los jóvenes riojanos prolonguen su permanencia o vida formativa en el sistema educativo.

\section{Protección y Prevención en la atención a la infancia}

Según el artículo I punto 2 la Ley I/2006, de 28 de febrero, de Protección de Menores de La Rioja, se entiende por protección de menores el conjunto de actuaciones que deben realizar las Administraciones Públicas con la finalidad de promover el desarrollo integral de los menores, garantizar sus derechos, proporcionarles la asistencia moral o material de la que carezcan, total o parcialmente, en su medio familiar y, en su caso, procurar su reeducación y reintegración social.

Esta misma ley, (Ley I/2006, de Protección de Menores de La Rioja) atribuye a la Consejería competente en servicios sociales una función directiva en esta materia, que comprende el desarrollo de políticas públicas que promuevan el conocimiento y respeto de

\footnotetext{
${ }^{15}$ De acuerdo con el Sistema estatal de indicadores de la educación 2014 (INEE, 20I4, p. 90) nos referimos al término "abandono temprano de la educación y formación" como el porcentaje de alumnos de i8 a 24 años que no siguen ningún tipo de educación o formación y que tienen como estudios máximos Educación Secundaria Obligatoria o anteriores niveles educativos. Una tasa alta de abandono escolar temprano se relaciona con unas consecuencias negativas y unos cotes elevados para los individuos y para la sociedad, tanto desde el punto de vista del mercado laboral como del bienestar en general.
} 
esos derechos en todos los ámbitos y el desarrollo con carácter prioritario de una política de prevención de situaciones de riesgo de protección infantil, a través de diferentes programas y recursos.

La protección integral del menor significa que deberán llevarse a cabo todas las intervenciones orientadas a promover el bienestar de los menores. En este sentido el capítulo que a continuación se presenta se centra en la atención a los menores en situación de dificultad.

Para analizar esta cuestión se realizó una Mesa de Expertos compuesta por técnicos de los principales programas y servicios de atención a menores de la Comunidad Autónoma de la Rioja. En este capítulo se presentan las principales conclusiones de la consulta realizada a los expertos y el debate en la sesión conjunta.

En el análisis de situación que se realizó desde cada una de las entidades participantes en la Mesa, se señalaron como principales problemáticas que afectan a los menores las siguientes:

Diferentes formas de violencia:

- Víctimas o autores de actos delictivos

— Niños Instrumentalizados en el conflicto familiar

— Violencia ejercida hacia los adultos: violencia filioparental.

Carencias de integración que a su vez generan dificultades respecto al derecho a la educación:

- Discriminación o segregación cultural /étnica

- Discapacidad

- Pobreza

La violencia aparece como uno de los temas que más preocupan, por la vulneración de derechos que implica padecer situaciones de violencia o ejercer violencia hacia otros. En este punto, es importante destacar que la vivencia de violencia genera pautas violentas en los menores. De ahí la importancia de detectar a tiempo las situaciones de violencia intrafamiliar. Los menores pueden ser también autores de infracciones, siendo de las que más preocupan la violencia entre iguales y la violencia de género en parejas jóvenes.

Asimismo, en el ámbito doméstico, los expertos alertan sobre la instrumentalización de los menores de edad en el conflicto familiar, particularmente en las separaciones contenciosas. Los hijos con frecuencia son testigos de las situaciones de violencia de género padecida por la madre y posteriormente son utilizados como instrumentos en la relación conflictiva generada tras la separación.

Una de las manifestaciones de la violencia que mayor desconcierto está causando entre todos los profesionales implicados en la atención a menores es la violencia filioparental. En este caso el niño pasa de ser víctima a ser agresor. Probablemente no sea un comportamiento nuevo en las relaciones familiares, aunque es recientemente cuando empieza a tomar dimensiones preocupantes entre las familias y los expertos. 
Se apunta también a la situación de los menores con alguna discapacidad y los problemas de integración derivados como consecuencia de la misma. Si bien, siendo un tema relevante en los procesos de inclusión social, quedó apuntado por parte de los expertos, aunque no fue suficientemente desarrollado, dada la composición de la Mesa. Si bien, resulta relevante destacar la identificación de esta situación como una dificultad que afecta a un sector de la población y por tanto, un tema a considerar como área de mejora.

La cuestión de la crisis y la de la pobreza y sus consecuencias en la integración de los menores también fue puesta de manifiesto como un problema que afecta a la protección de los menores. Los expertos identifican diversas manifestaciones de esta problemática, en algunos casos a través de dificultades para participar en actividades extraescolares; en otros para disponer del material para los estudios, por ejemplo ante la eliminación del programa de gratuidad de los libros de texto; y, en casos muy extremos, mediante intervenciones administrativas como la declaración de desamparo el internamiento de los menores en centros de acogida para garantizar la supervivencia de los menores, ante la ausencia de recursos económicos en la familia para atender las necesidades básicas del hogar. En la nueva ley de Protección de la Infancia recientemente aprobada esta situación estaría prohibida, si no existen otros factores de desprotección.

Los expertos aluden también al riesgo de sobreprotección y cronificación de determinadas familias en los dispositivos de ayuda. Esto tipo de situaciones llevan a la necesidad de revisar los sistemas de organización y la coordinación interinstitucional.

En suma, se puede afirmar que la crisis ha plasmado o agudizado problemas en los sistemas de protección social. Las medidas encaminadas a paliar este tema son a medio y largo plazo. Sin embargo la tendencia es atender lo urgente. Es responsabilidad de la Administración, como titular de obligaciones poner los medios adecuados para atender las situaciones de dificultad que afectan a los menores. De acuerdo a la Ley I/2006, corresponde a la Administración de la Comunidad Autónoma de La Rioja, a través de la Consejería competente en materia de Servicios Sociales, la declaración de las situaciones, la tramitación de los procedimientos y la adopción y ejecución de las medidas de protección que regula la Ley, así como la coordinación general de la atención a los menores, la planificación de la misma y la evaluación de los programas (artículo 3, punto I Ley I/2006). Para la ejecución de las medidas podrá habilitar a entidades colaboradoras especializadas en atención a menores.

\section{El derecho a la participación}

El derecho a la participación no está recogido de forma expresa en ningún artículo de la CDN. Si bien entre los artículos I2 y i7 se hace referencia a diferentes dimensiones relacionadas con la participación, tales como la libertad de expresión, de opinión, de pensamiento, de asociación, a estar informado, etc. De la suma de todos ellos se reconoce el Derecho a Participar ${ }^{16}$. Si bien en ninguno de ellos se utiliza expresamente el término participación. Sin embargo, si aparece en el artículo 23 en referencia a facilitar la

${ }^{16}$ Benito, A. (2006) La participación infantil en los programa de educación no formal, en González, R. (coord.) El derecho a la participación infantil de los niños, niñas y adolescentes en situación de riesgo. Ed. Comunidad de Madrid y Save the Children., disponible en internet

http://www.cse.coop/marta/pdf/Derecho_a_la_participaci\%C3\%B3n_infantil.pdf 
participación activa en la comunidad de los niños impedidos y en el artículo 3I, sobre participación en la vida cultural y artística ${ }^{\text {I7 }}$.

Este derecho debe interpretarse con relación al artículo i2 de la Convención, donde de forma resumida se puede concretar en los siguientes términos: "El niño tiene derecho a expresar su opinión y a que ésta se tenga en cuenta en todos los asuntos que le afectan".

Con el fin de clarificar el contenido del artículo i2, el Comité para los Derechos del Niño redactó en 2009, la Observación General $n^{\circ}$ I2 sobre el Derecho del niño a ser escuchado. En el punto 3 de la introducción al documento, se destaca que:

En los últimos años se ha ido extendiendo una práctica que se ha conceptualizado en sentido amplio como «participación», aunque este término no aparece propiamente en el texto del artículo 12. Este término ha evolucionado y actualmente se utiliza por lo general para describir procesos permanentes, como intercambios de información $y$ diálogos entre niños $y$ adultos sobre la base del respeto mutuo, en que los niños puedan aprender la manera en que sus opiniones y las de los adultos se tienen en cuenta $y$ determinan el resultado de esos procesos.

En este sentido, cabe afirmar que en el derecho de participación tan importante como el resultado es el proceso, dado el impacto que tiene el aprendizaje de la participación en la socialización de los niños y niñas, el reconocimiento de su condición de ciudadanos y su influencia en la vida adulta.

En el documento se describe cómo debe ser interpretado el derecho del niño a ser escuchado en diferentes ámbitos y situaciones que afectan a su vida cotidiana (familia, escuela, procesos de acogimiento, etc.). Entre las aportaciones de la Observación cabe destacar que hace referencia explícita a que este derecho alcanza tanto a cada niño individualmente, como de forma colectiva. Además señala que la participación no debe ser un acto momentáneo, sino el punto de partida de un intercambio de puntos de vista entre niños y adultos.

Un elemento específico dentro del derecho a la participación es el de participar en la vida cultural y artística. Este aspecto está recogido en el artículo 3I de la Convención. En el mismo se establece el reconocimiento, de los Estados Partes firmantes del mismo, al derecho de los niños y niñas al descanso y el esparcimiento, al juego y a las actividades recreativas propias de su edad y a participar libremente en la vida cultural y en las artes.

Asimismo destaca que los Estados Partes respetarán y promoverán el derecho de niños y niñas a participar plenamente en la vida cultural y artística y propiciarán oportunidades apropiadas, en condiciones de igualdad, de participar en la vida cultural, artística, recreativa y de esparcimiento.

En la Observación General número I7 sobre el citado derecho aprobada por el Comité de los Derechos del Niño en su $62^{\circ}$ periodo de sesiones (I4 de enero a I de febrero de 2013), el Comité muestra su preocupación por "el escaso reconocimiento que los Estados otorgan a los derechos contenidos en el artículo 31" (2013:3).

${ }^{17}$ GAITÁN, L. (20I4) De "menores" a protagonistas. Los derechos de los niños en el trabajo social, ed. Impulso a la acción social, Madrid, pág. I4I 
El comité señala en un único párrafo la importancia de este tipo de actividades en el desarrollo y socialización de la infancia.

Por su parte la Ley i/2006 de 28 de febrero de Protección de menores de la Rioja ${ }^{\mathrm{1}}$, dedica el artículo i7 al Derecho al juego, al ocio y la cultura. En el mismo se establece que:

I. Todo menor tiene derecho al juego, al ocio y a la participación activa en la vida cultural, deportiva, recreativa y artística de su entorno como elementos esenciales de su desarrollo evolutivo y proceso de socialización.

2. Las Administraciones Públicas de La Rioja promoverán y fomentarán la realización de actividades culturales, deportivas, artísticas y recreativas adaptadas a las necesidades de los menores y la participación de los mismos en dichas actividades.

En los reglamentos de desarrollo de la citada ley no se incluye ninguno específico para el derecho al juego, al ocio y la cultura. ${ }^{19}$ Por tanto, el instrumento de garantía del derecho debería articularse a través del III Plan Integral de Infancia (20II-20I4) aprobado por el Consejo de Gobierno el I de abril de 20 II.

Dicho Plan está organizado en cinco áreas. El Derecho a participar en la vida cultural y artística es únicamente abordado en la dimensión deportiva, quedando por tanto, insuficientemente reflejado en el mismo.

Por su parte, el Ayuntamiento de Logroño ha aprobado recientemente el Plan de Infancia para el periodo $2014-2018^{20}$. El plan ofrece un amplio análisis y diagnóstico de situación de nueve áreas temáticas, entre las que se incluye Cultura, Deporte y Ocio y tiempo libre. A partir del mismo establece objetivos y acciones en cinco áreas, siendo una de ellas Tiempo libre, cultura y deporte. La definición de los objetivos toma como punto de partida el artículo 3I de la Convención, y se plantean en los siguientes términos:

2.I. Ofrecer a las familias Servicios de Atención a la Infancia en el tiempo libre, a través de profesionales que apoyen la labor de los padres, poniendo a su disposición recursos que favorezcan el desarrollo de la personalidad, la socialización de los niños y niñas, así como la conciliación de la vida familiar y laboral.

2.2. Potenciar el desarrollo integral de la infancia a través del tiempo libre educativo y accesible, con una metodología educativa, participativa, flexible y personalizada, favoreciendo la educación en valores.

2.3. Desarrollar la creatividad infantil y la cultura a través de actividades lúdico educativas.

2.4. Facilitar la práctica deportiva entre todos los niños y adolescentes para favorecer su desarrollo personal y social.

\footnotetext{
${ }^{18} \mathrm{http} / /$ sid.usal.es/leyes/discapacidad/9006/3-2-I/ley-I-2006-de-28-de-febrero-de-proteccion-de-menoresde-la-rioja.aspx

${ }^{19}$ Afirmación realizada en base a la información disponible en el sitio web del Gobierno de la Rioja http://www.larioja.org/npRioja/default/defaultpage.jsp?idtab=809406\&modelo=NA\&materiaP=I9o\&mat eriaS $=08$ o\&materia $\mathrm{T}=\mathrm{T}$

${ }^{20}$ Consejo Municipal de la Infancia y la Adolescencia de Logroño celebrada en el Salón de Plenos Municipal del día I9 de diciembre de 2013.
} 
Como se puede ver el Plan municipal es más exhaustivo en la garantía del derecho a participar en la vida cultural y artística que lo establecido en el Plan del Gobierno. Este aspecto debería ser revisado en una futura edición del mismo.

\section{Recomendaciones principales}

En este último epígrafe se presentan las recomendaciones principales que, a la vista del Informe, se realiza desde UNICEF Comité de La Rioja como los desafíos principales para lograr el pleno cumplimiento de la Convención sobre los Derechos del Niño en el contexto de la Comunidad Autónoma de La Rioja.

I. Invertir los recursos económicos necesarios para garantizar la igualdad de oportunidades y la no discriminación de los niños y las niñas, especialmente en los ámbitos de la educación, la sanidad, los servicios sociales y la protección de menores, y aproximar así la inversión en infancia a la media europea.

2. Desarrollar mecanismos de mejora de la coordinación de todos los órganos de la administración que tienen funciones para con la infancia en la Comunidad de La Rioja. La vida de los niños más vulnerables trasciende los límites competenciales de un determinado programa o servicio y requiere la actuación integrada de los diferentes recursos.

3. Velar por la igualdad de oportunidades en el ejercicio del derecho a la educación, y adoptar medidas concretas para promover el éxito escolar que involucren a las administraciones, la escuela, la familia y el entorno educativo, reforzando así la adherencia del niño al proceso educativo.

4. En el ámbito de la protección de la infancia, impulsar y mejorar todo el sistema de protección autonómico, aumentando la inversión en recursos humanos, económicos y programáticos.

5. Impulsar el derecho a la participación y el empoderamiento ciudadano de los niños y niñas, a través de mecanismos explícitos que les permitan participar de todos los asuntos que les afectan. 